\title{
The tumor microenvironment and immune responses in prostate cancer patients
}

\author{
J T W Kwon1, R J Bryant1,2 and E E Parkes1 \\ 1Department of Oncology, University of Oxford, Oxford, UK \\ 2Nuffield Department of Surgical Sciences, University of Oxford, Oxford, UK
}

Correspondence should be addressed to E E Parkes: eileen.parkes@oncology.ox.ac.uk

This paper is part of a thematic review section celebrating 80 Years of Androgen Deprivation as a Treatment for Prostate Cancer. The guest editors for this section were Amina Zoubeidi and Paramita Ghosh.

\begin{abstract}
The landscape of cancer treatment has been transformed over the past decade by the success of immune-targeting therapies. However, despite sipuleucel-T being the firstever approved vaccine for cancer and the first immunotherapy licensed for prostate cancer in 2010, immunotherapy has since seen limited success in the treatment of prostate cancer. The tumour microenvironment of prostate cancer presents particular barriers for immunotherapy. Moreover, prostate cancer is distinguished by being one of only two solid tumours where increased T cell-infiltration correlates with a poorer, rather than improved, outlook. Here, we discuss the specific aspects of the prostate cancer microenvironment that converge to create a challenging microenvironment, including myeloid-derived immune cells and cancer-associated fibroblasts. By exploring the immune microenvironment of defined molecular subgroups of prostate cancer, we propose an immunogenomic subtyping approach to single-agent and combination immune-targeting strategies that could lead to improved outcomes in prostate cancer treatment.
\end{abstract}

\section{Introduction}

Prostate cancer (PCa) is the second most commonly diagnosed cancer and the fifth most common cause of death in men worldwide (Sung et al. 2021). Although the aetiology of PCa is multifactorial, genomics is a key variable underpinning the development of this malignancy. Metaanalysis of genome-wide association data depicted an increase in incidence of PCa in men of African ancestry and the estimated lifetime risk of developing $\mathrm{PCa}$ is compounded by a positive familial history (Conti et al. 2021). Whilst many cases of low-risk volume PCa may be managed by active surveillance and not need immediate treatment, clinically significant localised and locally advanced PCa may be treated with curative intent by surgery or radiotherapy with concomitant androgen deprivation therapy (ADT). However, despite earlystage disease having a 5-year relative survival rate of approximately $100 \%$, patient survival drops markedly to around 30\% once PCa has disseminated (Siegel et al. 2020). Furthermore, advanced-stage disease in most cases develops resistance to $\mathrm{ADT}$, underscoring the unmet clinical need to develop new treatments for metastatic castration-resistant PCa (mCRPC).

Although the effects of DNA repair defects and genetic/ epigenetic aberrations on the cell division machinery are increasingly well-defined, it has become evident that the tumour microenvironment (TME) plays a pivotal role (c) 2021 The authors Published by Bioscientifica Ltd. Printed in Great Britain

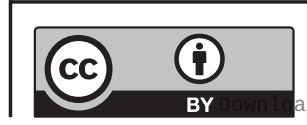

This work is licensed under a Creative Commons Attribution 4.0 International License. 
in the survival and propagation of tumours (Hinshaw \& Shevde 2019). The TME comprises supporting stromal cells, endothelial cells and immune cells, each of which contributes to cancer development and behaviour. Lifestyle factors, such as microbiota and diet, which play key roles in the TME, have also been associated with prostate tumourigenicity (de Bono et al. 2020). By better characterising the TME, in particular the immune populations resident within these niches, we can begin to elucidate the immunosuppressive circuits that facilitate immune evasion by cancer cells. Crucially, identifying such resistance mechanisms creates an opportunity for the use of immunotherapies in effectively 'switching on' the immune system to recognise and eradicate cancers.

There are currently two immunotherapies approved by the US Food and Drug Administration (FDA) for PCa treatment. Sipuleucel-T is an autologous active cellular immunotherapy approved by the FDA in 2010 for the treatment of asymptomatic or minimally symptomatic mCRPC. This first-in-class cancer vaccine utilises patientderived peripheral blood mononuclear cells (PBMCs) that have been stimulated ex vivo with a recombinant fusion protein consisting of prostatic acid phosphatase and granulocyte-macrophage colony-stimulating factor. Following leukapheresis and priming of antigen-presenting cells, the PBMCs are reinfused every 2 weeks for a total of three infusions. Results from the critical IMmunotherapy for Prostate AdenoCarcinoma Treatment (IMPACT) phase III study showed a significant improvement of 4.1 months in median overall survival (OS) in the treatment group compared to the placebo group. However, no significant difference was observed in median time to objective disease progression between the two patient cohorts (Kantoff et al. 2010).

The humanised monoclonal anti-PD-1 antibody, pembrolizumab, received accelerated approval from the FDA in 2017 for the treatment of unresectable or metastatic solid tumours. Of note, tumours needed to exhibit high microsatellite instability (MSI-H) or mismatch repair deficiency (dMMR). Supplementary to this molecular profile, requirements include disease progression following conventional therapy and a lack of viable alternative treatment options. Anti-PD-1 checkpoint blockade in the context of MSI-H/dMMR tumours is proposed to enhance prognosis by virtue of the hypermutated tumour phenotype possessing a greater neoantigen load and in turn, being associated with increased immune infiltration (Graham et al. 2020). Approval was predicated on the pooled analysis of patients enrolled across five clinical trials (KEYNOTE-012, -016, -028, -158 and -164) who were receiving pembrolizumab at either $10 \mathrm{mg} / \mathrm{kg}$ every 2 weeks or $200 \mathrm{mg}$ every 3 weeks. Data from 149 patients yielded an objective response rate of $39.6 \%$ (95\% CI: 31.7-47.9) with $78 \%$ of responses lasting 6 months or more (Marcus et al. 2019).

Both of these FDA-approved therapies have set important precedents in the immuno-oncology field. Sipuleucel-T was the first cancer vaccine and immunotherapy licensed for the treatment of PCa, while pembrolizumab was the first immunotherapy approved for a tumour-agnostic indication. Yet even with the advent of cancer vaccines and the success of immune checkpoint blockade (ICB) in other solid tumours such as melanoma, single-agent therapies have had limited success in the PCa setting (Kwon et al. 2014). This is thought to be due to the putative immunologically 'cold' nature of PCa, which tends to be characterised by restricted $\mathrm{CD}^{+} \mathrm{T}$ cell infiltration and low tumour mutational burden (TMB) (Alexandrov et al. 2013, Kaur et al. 2018).

Prostate tumours are also able to interact with supporting extracellular matrix and stromal elements to establish a chronic inflammatory and immunosuppressive milieu that is conducive to tumour growth. Herein, we review the PCa microenvironment and discuss immune subtypes typically present in PCa. Moreover, we propose a novel immunogenomic classification strategy to inform combination immunotherapy approaches for PCa.

\section{Immune cells in the prostate TME}

\section{T cells}

The inception of ICB has ushered in a new era of immunooncology where immune cells have replaced cancer cells as the principal focus of therapy. Of these immune cells, $\mathrm{T}$ cells, particularly cytotoxic $\mathrm{T}$ lymphocytes (CTLs), are most obviously vital during immune-mediated clearance of tumours (Van Allen et al. 2015). Tumour-infiltrating lymphocytes (TILs) are key mediators of anti-tumour immune responses and maintenance, with high TILs correlating with improved prognosis in breast cancer, colorectal cancer, melanoma and other solid organ malignancies (Mlecniketal. 2011, Loietal. 2019). This has led to the development of the 'Immunoscore', where immune infiltrate is quantified as a prognostic classification for solid tumours (Bruni et al. 2020). PCa, however, is distinguished by being one of only two tumour types (the other being renal cell carcinoma) where increased $\mathrm{CD}^{+} \mathrm{T}$ cell infiltration correlates with poor prognosis (Kaur et al. 2018,

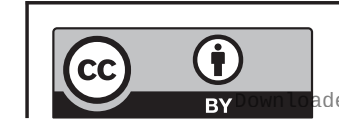

This work is licensed under a Creative Commons Attribution 4.0 International License. 
Petitprez et al. 2019). In these studies, PCa with increased CD8+ T cell density demonstrated earlier biochemical and metastatic relapse, and poorer OS. The reasons for this are currently unclear, with some data suggesting that highly $\mathrm{T}$ cell-infiltrated regions are associated with co-deletion of $B R C A 2$ and $R B 1$, which are typically features of highly aggressive, poor prognosis PCa (Calagua et al. 2021). The specific contribution of $\mathrm{T}$ cell infiltration to this prognostic outcome requires further characterisation. Interestingly, $\mathrm{T}$ cell infiltrated regions of PCa lymph node metastases have dysfunctional signalling patterns, with high expression of exhaustion markers such as PD-1 and TIM-3 (He et al. 2021). Although PCa is often described as immunologically 'cold', a subset of immune-infiltrated cases exists, but with distinct $\mathrm{T}$ cell signalling patterns. Therefore, a personalised approach to considering immunotherapy is required. This distinct poor prognostic role of TILs in PCa, in contrast with most solid tumours, may start to explain the limited success of T cell targeting therapies in PCa potentially even in immune-infiltrated cases.

Despite a minority of PCa cases presenting with increased $\mathrm{T}$ cell infiltration, the typical intra-tumoural microenvironment of PCa presents with reduced $\mathrm{T}$ cell density, especially in the CD8 ${ }^{+}$subset. This exclusion of $\mathrm{T}$ cells from the TME assists in forming an immune-privileged site (Philippou et al. 2020, Wu et al. 2020), with immune exclusion a key mediator of resistance to ICB (Mariathasan et al. 2018). Hypoxic cores, commonly present in PCa, are also devoid of $\mathrm{T}$ cell infiltrate and provoke aberrant angiogenesis programmes that cultivate neovasculature capable of impeding CTL extravasation (Motz et al. 2014). Therefore, even upon gaining access to the tumour, $\mathrm{T}$ cells are typically confronted by a highly suppressive PCa TME (Shalapour et al. 2015, Bezzi et al. 2018, Jayaprakash et al. 2018).

The organ specificity of the microenvironment may additionally bear importance. The bone is the most common metastatic site in PCa, and it is often the case that bone metastases are far less responsive to ICB than metastases situated in soft tissues (Beer et al. 2017, Halabi et al. 2016, Sharma et al. 2019). Bone resorption during osseous disease results in the release of sequestered transforming growth factor beta (TGFB), which together with interleukin 6 (IL-6) produced by bone marrow stromal cells, can act on resident T cells to preclude tumour killing (Jiao et al. 2019).

\section{B cells}

$\mathrm{T}$ cells are not the only immune population under consideration for mediating immunological treatment effectiveness. There is also a greater abundance of B cells in PCa compared to benign prostatic tissues, and the influx of B cells into the prostate TME has been linked with more aggressive disease (Woo et al. 2014, Wu et al. 2020). B cells, following their recruitment, can produce lymphotoxin, which activates IKKA-STAT3 and/or BMI1 signalling in residual cancer clones to hasten the onset of castration resistance and metastatic spread in PCa (Ammirante et al. 2010, 2013). In addition, IgA class-switched IL10+PD-L1+ plasma cells can attenuate CTL activation in a TGFBdependent manner, thereby hindering anti-tumour activity (Shalapour et al. 2015). More recently, however, greater plasma cell content in primary prostate tumours has been associated with increased inflammation and prolonged recurrence-free survival in men of African ancestry, an outcome that has been related to increased IgG expression and NK cell activity (Weiner et al. 2021). These findings allude to how differential expression of cytokines in the prostate TME may influence the anti-tumour immune response and could explain the discrepancy in the role of B cells during PCa progression.

\section{Macrophages}

A sizeable proportion of immune cells infiltrating PCa are from the myeloid lineage. Macrophages, traditionally dichotomised into either classical (M1) or alternative (M2) phenotypes, are one of the most prominent immune cell populations found within this malignancy. Their differing activation states can be achieved through various stimuli, IFNG or LPS in the case of the pro-inflammatory M1 phenotype and IL-4, -6, -13 or stromal cell-derived factor 1 (SDF-1) for the anti-inflammatory M2 phenotype (Comito et al. 2014, Di Mitri et al. 2019), but it is also possible for macrophages to be polarised through their effector functions, for instance efferocytosis promoting M2 polarity (Yang et al. 2015). Over-representation of M2 macrophages in prostatic tumours has been correlated with extracapsular extension and early biochemical relapse (Comito et al. 2014).

\section{Myeloid-derived suppressor cells}

Other protagonists in the TME immune suppression phenotype of PCa are myeloid-derived suppressor cells (MDSCs), with it now apparent that MDSCs are able to govern tumour resistance to therapy. In Pten ${ }^{\mathrm{PC}-/-}$, Myc-CaP and TRAMP-C1 allograft mouse models of PCa, MDSCs have been shown to be enriched in prostate tumours following surgical castration in a CXCR2-dependent

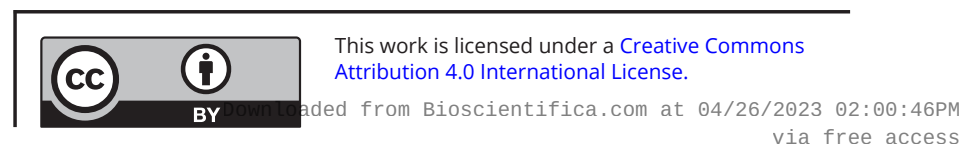


manner. Of note, this intra-tumoural immune infiltrate was able to confer resistance in PCa cells to ADT by secreting IL-23, attesting to the potential of immunotherapies as a means of enhancing current endocrine treatments for CRPC (Calcinotto et al. 2018). Through co-stimulatory interactions with MDSCs, mast cells can further enhance immunosuppression via engagement of CD40 and directly impairing CD8+ T cell function (Jachetti et al. 2018).

MDSCs are a particularly attractive target for therapy, considering their pervasiveness in PCa and their profound effect on T cell functionality. The extent of T cell infiltration in prostate tumours has been described to have an inverse relationship with the frequency of MDSCs. Eliminating the MDSC population could maximise the $\mathrm{T}$ cell load available within PCa to promote anti-tumour responses (Lu et al. 2017). To this end, Lu et al. demonstrated using a chimeric mouse model of mCRPC that ICB is able to synergise with the tyrosine kinase inhibitors cabozantinib and BEZ235 to overcome de novo resistance. Tyrosine kinase inhibition was able to potentiate the effects of ICB by downregulating multiple cytokines that promote the expression of immunosuppressive genes in MDSCs and by selectively depleting this immune cohort from the TME.

\section{Neutrophils}

Much like T helper $\left(\mathrm{T}_{\mathrm{h}}\right)$ cells and macrophages, neutrophils exhibit plasticity in their phenotype and can be polarised into anti-tumourigenic ' $\mathrm{N} 1$ ' or pro-tumourigenic 'N2' subtypes through various cytokines, including but not limited to G-CSF, IL-1B and TGFB (Fridlender et al. 2009, Casbon et al. 2015). Depletion of neutrophils in a mouse model of PCa metastasis augmented growth in bone, indicating a crucial role of this immune cell subset in promoting cancer growth. Furthermore, PCa appears to adjust to neutrophils over time with neutrophil cytotoxicity being gradually abrogated, correlating with prolonged neutrophil viability and diminished neutrophil extracellular trap formation (Costanzo-Garvey et al. 2020). These findings suggest that neutrophils may be able to combat the development of PCa metastases in bone, but caution must be exercised when devising therapies targeting these cells given the temporal dynamics of neutrophil effector function in the TME.

\section{Cancer-associated fibroblasts}

Although not an immune cell, cancer-associated fibroblasts (CAFs) are major players in the immunosuppressive circuitry of tumours. Crosstalk of CAFs with M2 macrophages promotes prostate carcinogenesis and further still, M2 macrophages themselves stimulate CAF development by eliciting epithelial-to-mesenchymal transition in PCa cells and trigger neovascularisation (Comito et al. 2014). Additionally, ADT can induce hypoxia, which triggers autocrine TGFB signalling and trans-differentiation of CAFs into CXCL13-producing myofibroblasts (Ammirante et al. 2014). This subset of CAFs then recruits IgA+ plasmacytes that consequently impede CTL activity (Shalapour et al. 2015). The communication between cancer, immune and stromal cells presents a tight-knit community of resistance to immune-targeting treatments in PCa that requires further investigation.

\section{Androgen deprivation therapy and immune responses in prostate cancer}

The androgen receptor is a crucial factor driving PCa progression, and anti-androgen therapies are a mainstay of treatment. ADTs reduce circulating testosterone by inhibiting gonadotrophin releasing hormone, inhibiting the androgen receptor or androgen synthesis via inhibition of CYP17 (Desai et al. 2021). The effect of both testosterone and androgen deprivation on the systemic and prostate immune responses is reviewed in (Gamat \& McNeel 2017, Ben-Batalla et al. 2020) - ADT induces alterations in circulating $\mathrm{T}$ cells, increasing naïve and $\mathrm{T}_{\mathrm{h}} 1$ cells with a concomitant reduction in $\mathrm{CD}^{+}$ $\mathrm{T}$ regulatory cells $\left(\mathrm{T}_{\text {regs }}\right)$. Studies in mouse models indicate direct immunomodulatory effects of ADT on the PCa TME; however, depending on the model used (Pten ${ }^{-/-}$ or MYC-driven, for example) distinct effects have been observed. A study of clinical samples of matched preand post-ADT early-stage PCa treated with neoadjuvant ADT indicated that treatment resulted in upregulation of immune checkpoints, such as PD-1 and CTLA-4, as well as infiltration of immune cells, including CD8 ${ }^{+}$and $\mathrm{T}_{\mathrm{h}} 1$ cells (Long et al. 2020). However, this relatively small study (six matched biopsies) was unable to delineate the effects of distinct PCa subgroups in early stage disease.

Combination ADT-immunotherapies are now in clinical trials for mCRPC, with promising results from early phase studies being investigated in large phase III studies, for example studying enzalutamide in combination with anti-PD-1 compared to enzalutamide with placebo (KEYNOTE-641 (Graff et al. 2021)). However, a study of enzalutamide in combination with anti-PD-L1 did not 
result in improved OS in mCRPC compared to enzalutamide alone (IMbassador250, Sweeney et al. 2020). Interestingly, a detailed single cell RNA-seq study using pre- and postenzalutamide biopsies identified clonal expansion of $\mathrm{T}$ cells suggesting that response to enzalutamide (and tumour cell death) may be a pre-requisite for response to enzalutamide/anti-PD-L1/1 combination therapy (He et al. 2021). Additionally, although ADT is used across prostate adenocarcinomas, regardless of genomic subtype, it is not known if distinct subgroups of human PCa have differential immune responses to ADT. Moreover, the current role of ADT in combination immune therapy remains unclear, with further studies needed to determine the optimal combination of immune-targeting agents that synergise with ADT. The potential immunotherapeutic combination approaches in MCRPC is now an area of great clinical need, with approximately 41 ongoing clinical trials studying checkpoint inhibitor combinations in PCa (Bansal et al. 2021).

\section{Prostate cancer TME}

When exploring the tapestry of the TME (Fig. 1 and Table 1), we need to consider the net effect of immune cells on carcinogenesis along with ways in which we can shift the balance of effector functionality in these resident populations to reinstate anti-tumour immunity. The complexity of the suppressive immune populations present in PCa and the role of the TME in promoting cancer growth indicate the multiple factors requiring consideration in developing novel immunotherapy approaches. The current immunotherapy trials in PCa have been reviewed extensively elsewhere (Fay \& Graff 2020). However, the low tumour mutational burden of PCa and the limited success of current ICB strategies suggest a need for stratification of PCa for future therapies. Therefore, a combined immunogenomic classification may enable the selection of patients for tailored combination strategies.

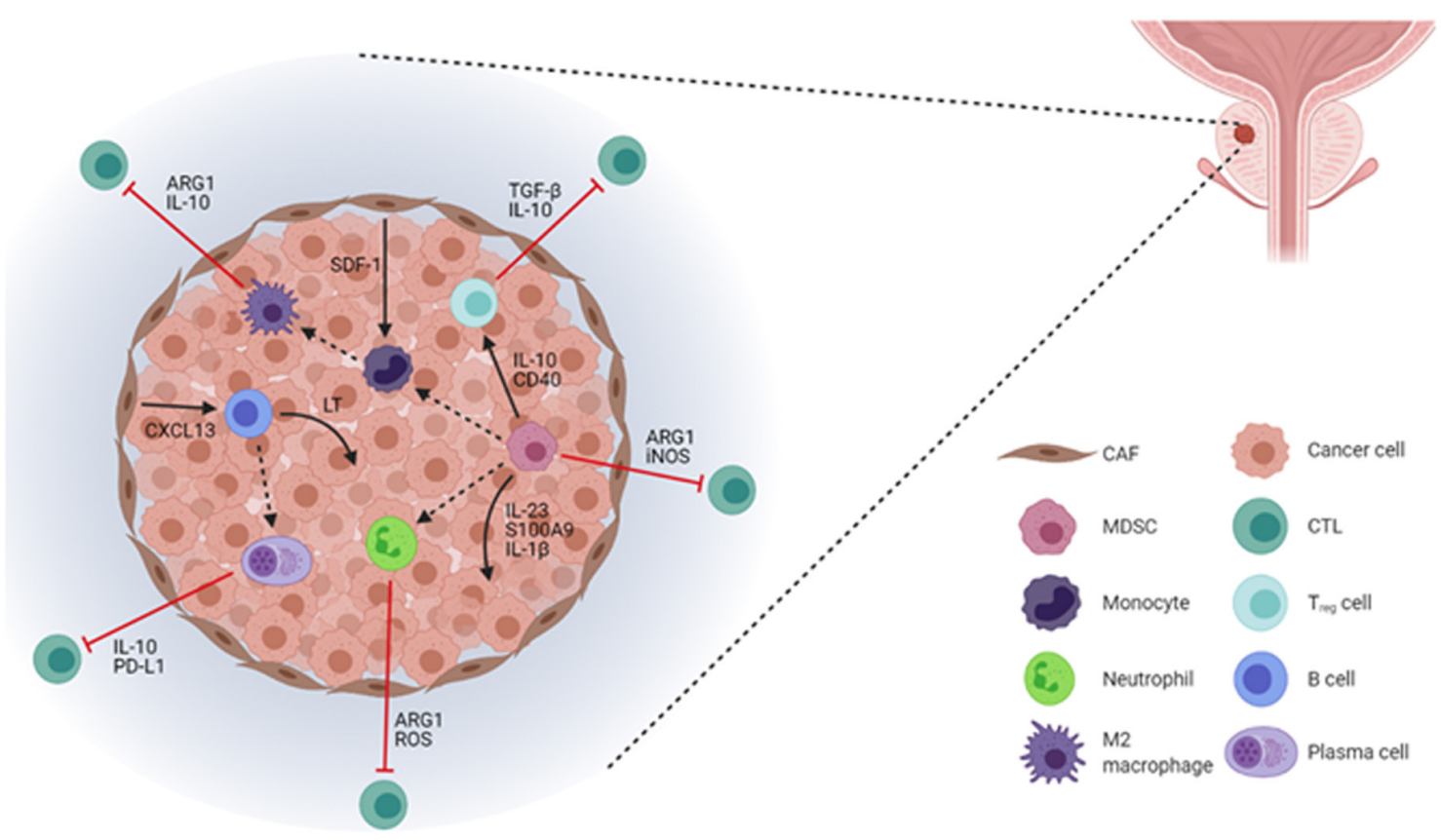

Figure 1

Immunosuppression and tumour-promoting circuitry within the prostate TME. Tumours are able to co-opt immune populations through various chemokine axes and initiate a complex interplay that facilitates tumour development. In addition to driving proliferation and survival of cancer cells through several soluble mediators, MDSCs upregulate suppressive factors that curb CTL activity while also being able to impede tumour killing through the recruitment of $\mathrm{T}_{\text {reg }}$ cells. Moreover, immature MDSCs can differentiate into either neutrophils or monocytes and subsequently M2 macrophages, all of which can further suppress CTL effector function. CAFs can additionally influence tumour immune contexture by recruiting B cells which not only support tumour growth and progression but also differentiate into immunosuppressive plasma cells. Alternatively, CAFs can instigate the differentiation of monocytes into M2 macrophages to sustain CTL inhibition. CAF, cancer-associated fibroblast; MDSC, myeloid-derived suppressor cell; CTL, cytotoxic T lymphocyte; LT, lymphotoxin; SDF-1, stromal cell-derived factor 1; ROS, reactive oxygen species; iNOS, inducible nitric oxide synthase; PD-L1, programmed death-ligand 1; S100A9, S100 calcium-binding protein A9.

https://erc.bioscientifica.com https://doi.org/10.1530/ERC-21-0149 (c) 2021 The authors Published by Bioscientifica Ltd. Printed in Great Britain

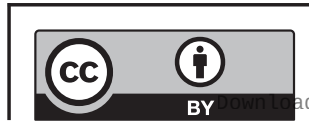

This work is licensed under a Creative Commons Attribution 4.0 International License. ded from Bioscientifica.com at $04 / 26 / 2023$ 02:00:46PM 
Table 1 Immune cells in the prostate cancer microenvironment.

\begin{tabular}{l}
\hline Cell type \\
\hline CAF \\
$\mathrm{T}_{\text {reg cell }}$ \\
B cell
\end{tabular}

\section{Pro-tumourigenic activity}

Trigger differentiation of monocytes into M2 macrophages (Comito et al. 2014); recruitment of B cells into TME (Ammirante et al. 2010, 2014) Inhibition of CTL effector function (Toso et al. 2014, Bezzi et al. 2018, Vidotto et al. 2019)

B cell Promote growth and survival signalling in cancer cells (Ammirante et al. 2010, 2013); differentiation into plasma cells (Shalapour et al. 2015)

Plasma cell MDSC Inhibition of CTL effector function (Shalapour et al. 2015)

Promote growth and survival signalling in cancer cells (Bezzi et al. 2018, Calcinotto et al. 2018); recruitment of Treg cells into TME; differentiation into monocytes or neutrophils; inhibition of CTL effector function (Toso et al. 2014, Lu et al. 2017, Bezzi et al. 2018)

M2 macrophage Inhibition of CTL effector function (Bezzi et al. 2018, Di Mitri et al. 2019)

Neutrophil Inhibition of CTL effector function (Fridlender et al. 2009, Casbon et al. 2015, Costanzo-Garvey et al. 2020)

\section{Immunogenomic subtypes of prostate cancer}

As demonstrated in various solid tumours, the genomic characteristics of the tumour have a profound influence on the TME. While our proposed classification is not exhaustive, it provides a framework for considering personalised immunotherapy for PCa (Fig. 2).

\section{PTEN-deficient prostate cancer}

Phosphatase and tensin homolog (PTEN) is one of the most commonly inactivated tumour suppressor genes in PCa, with around $20 \%$ of primary prostate tumours exhibiting aberrations in PTEN, rising to approximately $40 \%$ of mCRPC cases (Robinson et al. 2015). Somatic defects in PTEN are typically biallelic loss, with other inactivating point mutations, gene rearrangements and epigenetic changes occurring less frequently (Robinson et al. 2015). PTEN has a diametrically opposed role to phosphoinositide 3-kinase (PI3K), whereby it impedes signal transduction of the PI3K - Rac-alpha serine/threonine-protein kinase (AKT) - mechanistic target of rapamycin (mTOR) axis. As such, loss-of-function in PTEN in conjunction with amplifications and activating mutations in the PI3K pathway can synergise to promote higher-grade, more invasive PCa and tumour recurrence following treatment (Robinson et al. 2015). However, it is now becoming clear

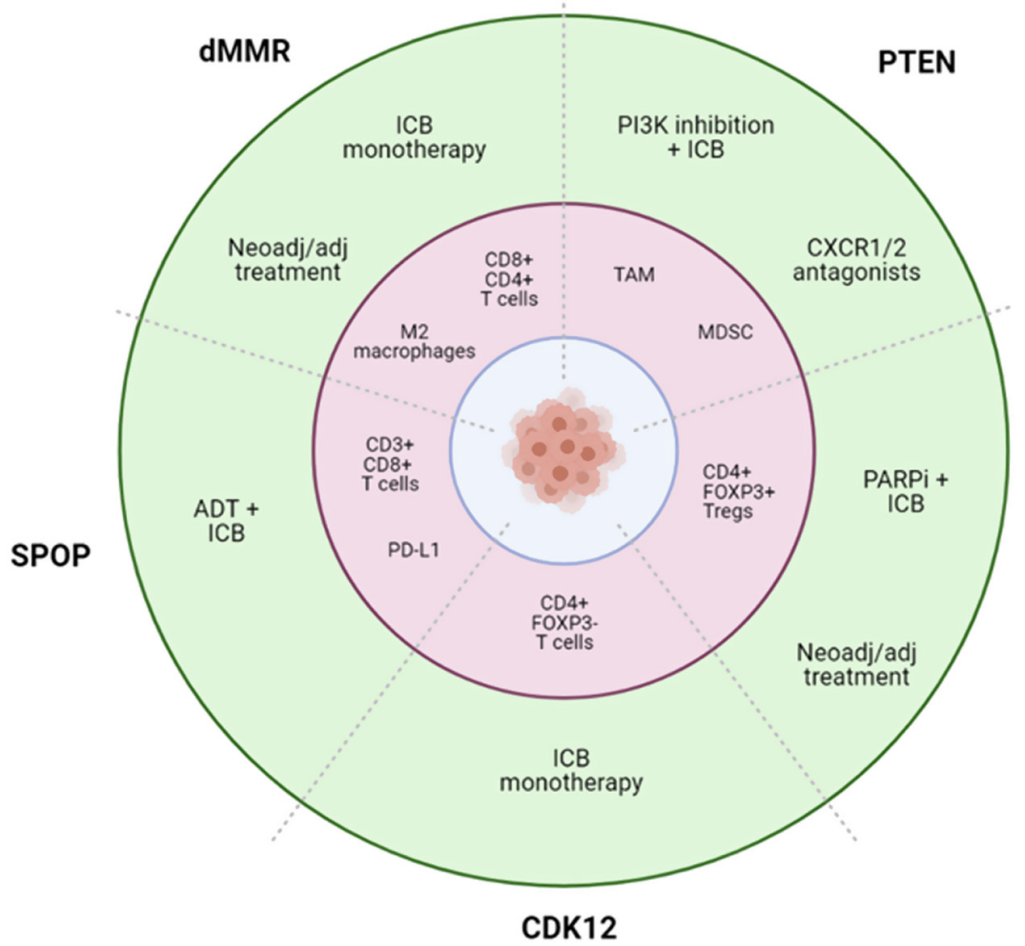

\section{Figure 2}

Immunogenomic subgroups and

immunotherapeutic treatment strategies for PCa. Five immunogenomic subgroups of PCa are described. The inner ring (red) indicates the immune infiltrate characterised in each subgroup to date. Distinct immune populations are present in different genomic subtypes of $\mathrm{PCa}$, indicating individual immune microenvironments to consider when designing immunotherapeutic treatment approaches. The outer ring (green) indicates potential treatment strategies for each subgroup. dMMR, microsatellite unstable/ mismatch repair-deficient; PTEN, PTEN-deficient; HRD, homologous recombination-deficient; CDK12, CDK12-mutated; SPOP, SPOP-mutated; $A D T$, androgen deprivation therapy; ICB, immune checkpoint blockade. https://erc.bioscientifica.com https://doi.org/10.1530/ERC-21-0149 (c) 2021 The authors Published by Bioscientifica Ltd. Printed in Great Britain
This work is licensed under a Creative Commons Attribution 4.0 International License.

ded from Bioscientifica.com at $04 / 26 / 2023$ 02:00:46PM 
that the function of PTEN extends beyond its role in antagonising PI3K signalling to modulating inflammation and immunity (Li et al. 2016).

Several pre-clinical studies have revealed that deficient PTEN expression facilitates tumour development and resistance to therapy through the recruitment of MDSCs (Calcinotto et al. 2018, Zhao et al. 2020). Interestingly, concomitant deletion of certain genes altered the balance of immune cells subsisting within PCa. $P t e n^{P C-/-} ; Z b t b 7 a^{P C-/-}$ tumours predominantly recruited polymorphonuclear-MDSC-like cells, which evoked tumour-promoting effects by stimulating NFKB signalling (Bezzi et al. 2018). Pten ${ }^{P C-1-}$; Trp53 $3^{P C-/-}$ tumours meanwhile recruited monocytic-MDSCs to enhance tumour growth by recruiting immunosuppressive $\mathrm{T}_{\text {regs }}$ and $\mathrm{M} 2$ macrophages. The latter mechanism of adaptive immune resistance was corroborated in another study where PTEN deficiency biased the PCa TME contexture towards greater FOXP3 ${ }^{+} \mathrm{T}_{\text {reg }}$ numbers and overexpression of indoleamine 2,3-dioxygenase 1 (Vidotto et al. 2019). This chronic inflammatory and highly immunosuppressive state cultivated by MDSCs likely accounts for the dampened anti-tumour activity in the TME, even in the presence of cytotoxic effector T cells (Kaur et al. 2018). The same principles could be applied to the refractory nature of PTENdeficient $\mathrm{PCa}$ to ICB, though an alternative explanation may be the 'immune desert' phenotype recapitulated, for example, by $\mathrm{Pten}^{\mathrm{PC}-/-}$; $P m l^{P C-/-}$ tumours (Bezzi et al. 2018). Moreover, prostate-specific deletion of Chd1 or Erk5 on a Pten ${ }^{\text {null }}$ background improved OS in mice relative to that solely lacking Pten expression; this prolongation in survival was attributed to curtailed chemoattraction of polymorphonuclear-MDSCs, thus permitting increased T cell infiltration into the TME (Zhao et al. 2017, 2020). As discussed above, therapies targeting MDSCs are promising in PTEN-deficient PCa, and are under clinical investigation in PCa more broadly.

Complete inactivation of PTEN is not only responsible for immunosuppression in the TME but also triggers senescence as a failsafe mechanism to counter prostate tumourigenesis (Chen et al. 2005). Several studies have reported that depletion of PTEN in PCa correlates with augmented expression of CXCL molecules, including CXCL1, CXCL2, CXCL5 and CXCL8 (Maxwell et al. 2013). The ensuing migration of monocytes/macrophages and myeloid cells via CXCR1/2-mediated chemotaxis into the TME subsequently promotes tumour progression, whether that be through initiating NFKB pro-survival signalling in tumour cells or by facilitating their escape from senescence (Armstrong et al. 2016). Pharmacological inhibition of CXCR2 was able to drive macrophages towards a pro-inflammatory state that induced growth arrest and senescence, specifically in prostate tumours deprived of PTEN expression (Di Mitri et al. 2019). These data demonstrate that blockade of CXCL-CXCR axes could serve as a viable treatment strategy for treating PCa patients that have been stratified according to PTEN status. However, caution should be exercised when leveraging senescence as a potential therapy approach. It has been evidenced that PTENnull prostate tumours, through NFKB and JAK2/STAT3 signalling, can give rise to a senescenceassociated secretory phenotype (SASP) geared towards the recruitment of MDSCs rather than lymphocytes (Toso et al. 2014). Indeed, Gr-1+ MDSCs have been shown to render the TME more permissive to tumour progression by antagonising the onset of senescence in prostate tumour cells (Di Mitri et al. 2014). PTEN-loss-induced senescence however does present opportunities for treatment - elimination of STAT3 signalling reconfigured the secretome of $\mathrm{Pten}^{\mathrm{PC}-/-}$ senescent $\mathrm{PCa}$ to reactivate immune surveillance and heighten clearance of senescent tumour cells (Toso et al. 2014). Of note, abrogating the JAK2/STAT3 axis strengthened the pro-senescent effects of docetaxel on PTEN-deficient PCa and a similar synergy was observed when docetaxel treatment was combined with a CXCR2 antagonist (Di Mitri et al. 2014). Furthermore, ablating inhibitor of apoptosis protein (IAP) signalling sensitised PTEN ${ }^{\text {null }}$ prostate tumours to clinically relevant doses of ionising radiation (Armstrong et al. 2016). Such observations delineate the therapeutic utility of abolishing tumour cell-intrinsic pathways to bolster anti-tumour immunity and overcome chemo-/radioresistance afforded by PTEN deficiency in PCa.

Prostate tumours additionally partake in crosstalk with their encapsulating stroma in order to remodel the TME and achieve immune subversion. CXCL8 stemming from PTEN deficiency has been demonstrated to both augment autocrine CXCR4/7 and CCR2 expression in human PCa cells and instigate the paracrine release of their cognate ligands, CXCL12 and CCL2, by prostate stromal fibroblasts and monocytes (Maxwell et al. 2014). As well as upholding the survival and expansion of PCa, stromalderived chemokines are likely involved in the recruitment and polarisation of other immune cell types that sustain tumour growth in the TME. A similar effect was observed in human PCa cells devoid of PTEN expression, where dysregulated AKT signalling enhanced CXCL12/CXCR4 expression by these cells and consequently amplified their proliferative and invasive capacities (Conley-LaComb et al. 2013). In models of bony metastases, stromal chemokines 
were able to induce AKT signalling in PCa cells and thus generate an osteolytic reaction in vivo that worsened tumour burden, perhaps by liberating immunomodulatory factors contained within the bone. Other studies demonstrated that loss of PTEN increased the expression of early growth response-1 (EGR1), a regulator of angiogenesis and osteoclast formation, across various PCa models ( $\mathrm{Li}$ et al. 2019).

PTEN deficiency, therefore, has a role in modifying tumour niches to favour tumour development and this may, in part, explain the propensity that advanced PTEN-deficient prostate tumours have for the bone microenvironment. At the same time, loss of PTEN appears to relieve control on tumour progression and metastasis, giving a rationale to target the PI3K-AKT and chemokine axes when treating PCa. Combining PI3K-AKT targeting with immune checkpoint blockade, therefore, represents a potential immune-sensitising approach in this subgroup, with pre-clinical modelling of this strategy supporting the ability of PI3K inhibition to relieve PTENdriven immunosuppressive activity and enhance immune responses (Qi et al. 2020).

\section{SPOP loss in prostate cancer}

Speckle-type pox virus and zinc finger protein (SPOP) missense mutations resulting in functional loss of this tumour suppressor occurs in approximately $10-15 \%$ of PCa (Blattner et al. 2017). Similar to PTEN loss, SPOP mutations promote PI3K-mTOR signalling with subsequent enhanced cancer cell proliferation and growth. However, SPOPmutated PCa appears to be a distinct genomic subgroup, co-occurring with CHD1 deletions, and is associated with responsiveness to $\mathrm{ADT}$, even in the metastatic setting (Swami et al. 2020). Interestingly, SPOP mutations result in increased PD-L1 levels in PCa, through the prevention of proteasome-mediated degradation (Zhang et al. 2018). This increased PD-L1 expression is closely associated with restriction of $\mathrm{CD}^{+}$and $\mathrm{CD} 8^{+} \mathrm{T}$ cell infiltration, promoting an immunosuppressive TME. Treatment of mouse models of Spop-mutant PCa with anti-PD-L1 resulted in responses, slowing tumour growth to the rate observed in wild-type Spop models.

The immune microenvironment of $S P O P$-mutant $\mathrm{PCa}$, however, remains relatively uncharacterised in terms of myeloid lineage cells, which, as discussed above, are key mediators of immunoresistance in PCa. Additionally, the typically high levels of sensitivity to ADT in this subgroup mean that careful consideration is needed to determine the optimal combination immunotherapy approach. ADT itself modulates the TME, as discussed above, with upregulation of genetic modules associated with antigen presentation, immune checkpoint genes and IFN- $\gamma$ signalling (Long et al. 2020). Similar to radiotherapy and chemotherapy, ADT is imprecise in terms of modulating immune responses, resulting in upregulation of both proand anti-tumourigenic immune pathways, which may indeed be PCa subtype-dependent. However, combination treatment with ADT and anti-PD-1 has shown promise in hormone-sensitive PCa, with single cell RNA-seq studies supporting the ability of this approach to optimise antitumourigenic immune responses (Hawley et al. 2020). Given the association of $S P O P$-mutant PCa with both ADT responsiveness and PD-L1 upregulation, this subgroup of PCa could be a promising target for this strategy.

\section{Mismatch repair-deficient prostate cancer}

Around $3-12 \%$ of PCa can be classified as having dMMR with somatic and germline pathogenic alterations in MMR genes (Abida et al. 2019). Studies have suggested that dMMR PCa is associated with a higher Gleason score (79\% Gleason 8-10) and metastatic disease (Graham et al. 2020). This is in contrast to colorectal cancer, where dMMR is associated with a good prognosis (Zaanan et al. 2018). This further highlights the unique factors of the PCa TME that need to be considered in optimising immunotherapy for this disease. Defects in the MMR pathway perturb the fidelity of the DNA replication process and give rise to genomic instability that mostly manifests within microsatellite regions. dMMR has therefore emerged as a predictive biomarker for ICB as the hypermutability associated with dMMR will increase the frequency of neoantigens capable of eliciting anti-tumour immunity. Studies of advanced PCa have shown that, of the dMMR mCRPC patients evaluable for objective responses, approximately 50\% demonstrated durable biochemical responses with PSA reduction, and a similar proportion displayed no signs of radiographic progression following PD-1/PD-L1 blockade (Abida et al. 2019, Graham et al. 2020). Although responders tend to derive clinical benefit from continued treatment with ICB, some non-responders with dMMR status experienced deterioration in their condition post-treatment with anti-PD-1/PD-L1, suggesting there are other underlying mechanisms that govern response to ICB.

In keeping with dMMR in other solid tumours, most dMMR PCa demonstrate increased infiltration of $\mathrm{CD}^{+}$and $\mathrm{CD}^{+}{ }^{+} \mathrm{T}$ cells, with increased expression of the

This work is licensed under a Creative Commons Attribution 4.0 International License. ed from Bioscientifica.com at 04/26/2023 02:00:46PM 
immune checkpoint PD-L1, although notably a significant proportion of dMMR PCa (approximately $45 \%$ based on a small study of nine cases) do not demonstrate increased lymphocytic infiltration, potentially accounting for lack of response to ICB in some cases. In the same study, gene expression analysis suggested increased infiltration of M2-polarised macrophages (Nava Rodrigues et al. 2018). However, considering the overall positive response rates to ICB, and with pembrolizumab now FDA-approved for this subgroup of $\mathrm{PCa}$, it is important to ensure timely testing of MSI status.

There is an argument for moving MSI testing to an earlier timepoint in the disease course - particularly given the success of neoadjuvant immunotherapy in the dMMR colorectal cancer setting (Chalabi et al. 2020). Immunotherapy in the neoadjuvant setting uses the tumour antigen load to encourage systemic long-lasting immune responses, targeting disease at a lower tumour burden. Taking this neoadjuvant approach in dMMR PCa is tantalising, offering a potential definitive first-line treatment for otherwise high-risk disease, although given the relatively low frequency of this subtype this would require an extensive testing strategy, and potentially optimising liquid biopsy companion diagnostic testing. A clinical trial investigating this approach could also explore potential biomarkers to identify patients who may benefit most from this strategy. A window study including combination (anti-CTLA-4 and anti-PD-1) or single-agent anti-PD-1 therapy could address the optimal approach for this subgroup. A platform study on this basis could also identify optimal immunotherapy combinations for dMMR PCa.

\section{Homologous recombination-deficient prostate cancer}

Deficiency of homologous recombination DNA repair, required for faithful repair of double-strand DNA breaks arising as a result of stalled DNA forks or crosslinked DNA, occurs in approximately $5-8 \%$ of early-stage $\mathrm{PCa}$, rising to $20-25 \%$ in the metastatic setting (Mateo et al. 2020). Similar to homologous recombination-deficient (HRD) ovarian and pancreatic cancers, $\mathrm{PCa}$ with mutations in key HR genes (including BRCA1/2, PALB2, CHEK1/2, and $A T M$ ) demonstrate sensitivity to poly(ADP-ribose) polymerase (PARP) inhibitors. In a landmark study, 711 patients were selected for molecular screening with 92 patients with metastatic PCa ultimately receiving the PARP inhibitor olaparib and being evaluable for response (Mateo et al. 2020). The highest response rates were observed in BRCA1/2-mutant PCa, and overall $57 \%$ of patients receiving the higher olaparib dose $(400 \mathrm{mg})$ demonstrated clinical responses.

Treatment with PARP inhibition results in upregulation of interferon signalling, via activation of the STimulator of INterferon Genes (STING) pathway and promoting immune cell infiltration, and also increased immune checkpoint (PD-L1) expression (Vikas et al. 2020). Thus, the combination of PARP inhibition and ICB is a logical approach in HRD PCa, with a number of trials of this combination ongoing in mCRPC, with some evidence that this approach may even be effective in HR-proficient PCa (Karzai et al. 2018).

Even in the early stages, HRD PCa is an aggressive subtype, associated with a poor outcome following radical radiotherapy and moderate resistance to docetaxel (Taylor et al. 2017). BRCA2-mutant $\mathrm{PCa}$, in particular, is associated with younger age at diagnosis of PCa, along with increased rates of both nodal and distant metastasis and a poorer prognosis. As above, it has been suggested that HRD PCa may be associated with increased lymphocytic infiltration, characterised by a $\mathrm{CD}^{+}{ }^{+} \mathrm{FOXP3}^{+} \mathrm{T}_{\text {regs }}$ intratumoural infiltrate and an increased CD8 $+\mathrm{T}$ cell infiltrate (Jenzer et al. 2019). A non-significant increase in $\mathrm{CD} 163^{+}$macrophages was also noted. This highly infiltrated immune TME alongside a dismal outlook for this subgroup of PCa justifies consideration of immune-targeting therapies at an earlier stage. Moreover, detailed characterisation of the TME in HRD PCa is required to select appropriate immunetargeting therapies. Given the low frequency of HRD in early stage $\mathrm{PCa}$, a consortium approach could identify these tumours at an early stage, thereby permitting an appropriately powered study of the single cell immune landscape of HRD PCa and identification of appropriate immune strategies. A further limitation in this field is the lack of HRD in vivo models for PCa, and better preclinical models would enable in-depth immune analysis of this subgroup.

\section{CDK12-mutated prostate cancer}

Although implicated in homologous recombination, cyclin-dependent kinase 12 (CDK12)-mutated $\mathrm{PCa}$ is genomically and immunologically distinct from HRD PCa, with limited PARP inhibitor responsiveness, high levels of genomic rearrangements, high neoantigen burden (second only to dMMR PCa) and increased lymphocytic infiltration (Antonarakis et al. 2020, Rescigno et al. 2021). In a large study of mCRPC, $4.7 \%$ were found to harbour CDK12 
alterations, associated with poorer OS. Characterisation of the TME by transcriptomic and immunohistochemical analysis demonstrating increased $\mathrm{CD}^{+} \mathrm{T}$ cell infiltration, with CD4+ FOXP3- immunosuppressive $\mathrm{T}$ cells being the dominant population (Rescigno et al. 2021). Consistent with this observation, a small study of 9 patients receiving anti-PD-1 ICB demonstrated a 33\% response rate, with $56 \%$ of patients remaining on treatment beyond 6 months (Antonarakis et al. 2020). These findings justify further characterisation of the myeloid compartment of these tumours, as well as investigation of CDK12 alterations in forthcoming studies of ICB in PCa as a biomarker for stratification for single-agent ICB treatment.

\section{Conclusions}

It is clear that PCa comprises more than one entity, with a number of immunogenomic subsets that could be exploited for therapeutic benefit using immunotherapy. A 'one-size-fits-all' approach is unlikely to result in successful clinical regimens, and consideration of the best approaches for detecting these subgroups is now needed in order to enable stratification for immunotherapeutic treatment. To that end, mining of clinical, genomic, transcriptomic and available histopathological data from existing trials of immunotherapy in PCa could enable determination of the appropriate subgroups for either single-agent ICB or specific combination strategies.

\section{Future directions}

The discussions above demonstrate the principle of offering an immune-targeting treatment at the earliest possible stage of disease. The combination of heavily pretreated disease, increased heterogeneity and established immunosuppressive networks in advanced metastatic PCa makes this a challenging population for immune-targeting treatments. Potential future clinical studies could focus on neoadjuvant window studies, using surrogate markers of response such as immune infiltration and detailed characterisation of immune responses, or de novo metastatic disease with lower tumour burdens. Early testing of tumours to detect, in particular, MSI or HRD could enable patients with these subgroups of disease to receive more aggressive immune-targeted treatment at an earlier stage, increasing the probability of long-term control and potential cure. The immunogenomic subgroups we propose above could be further stratified according to early or late-stage disease, and umbrella clinical trial designs incorporating ongoing biomarker discovery and validation used to select the appropriate immune combination treatments for each subgroup. This personalised immunotherapy approach could radically transform the future clinical outlook for men with PCa.

\section{Declaration of interest}

The authors declare that there is no conflict of interest that could be perceived as prejudicing the impartiality of this review.

\section{Funding}

J T W K, R J B and E E P are supported by funding from Prostate Cancer UK (Major Award in Immunology). R J B is supported by a Cancer Research UK/Royal College of Surgeons of England Clinician Scientist Fellowship (reference C39297/A22748). E E P is supported by a personal fellowship (Young Investigator Award) from the Prostate Cancer Foundation (2019).

\section{Author contribution statement}

J T W K and E E P conceptualised the framework for this review. All authors contributed to writing and editing.

\section{References}

Abida W, Cheng ML, Armenia J, Middha S, Autio KA, Vargas HA, Rathkopf D, Morris MJ, Danila Slovin SF, et al. 2019. Analysis of the prevalence of microsatellite instability in prostate cancer and response to immune checkpoint blockade. JAMA Oncology 5 471-478. (https:// doi.org/10.1001/jamaoncol.2018.5801)

Alexandrov LB, Nik-Zainal S, Wedge DC, Aparicio SAJR, Behjati S, Biankin AV, Bignell GR, Bolli N, Borg A, Børresen-Dale AL, et al. 2013 Signatures of mutational processes in human cancer. Nature $\mathbf{5 0 0}$ 415-421. (https://doi.org/10.1038/nature12477)

Ammirante M, Luo JL, Grivennikov S, Nedospasov S \& Karin M 2010 B-cell-derived lymphotoxin promotes castration-resistant prostate cancer. Nature 464 302-305. (https://doi.org/10.1038/nature08782)

Ammirante M, Kuraishy AI, Shalapour S, Strasner A, Ramirez-Sanchez C, Zhang W, Shabaik A \& Karin M 2013 An IKK $\alpha-E 2 F 1-B M I 1$ cascade activated by infiltrating B cells controls prostate regeneration and tumor recurrence. Genes and Development 27 1435-1440. (https://doi. org/10.1101/gad.220202.113)

Ammirante M, Shalapour S, Kang Y, Jamieson CAM \& Karin M 2014 Tissue injury and hypoxia promote malignant progression of prostate cancer by inducing CXCL13 expression in tumor myofibroblasts. PNAS 111 14776-14781. (https://doi.org/10.1073/ pnas.1416498111).

Antonarakis ES, Isaacsson Velho P, Fu W, Wang H, Agarwal N, Sacristan Santos V, Maughan BL, Pili R, Adra N, Sternberg CN, et al. 2020 CDK12-altered prostate cancer: clinical features and therapeutic outcomes to standard systemic therapies, poly (ADP-ribose) polymerase inhibitors, and PD-1 inhibitors. JCO Precision Oncology 4 370-381. (https://doi.org/10.1200/po.19.00399)

Armstrong CWD, Maxwell PJ, Ong CW, Redmond KM, McCann C, Neisen J, Ward GA, Chessari G, Johnson C, Crawford NT, et al. 2016 
PTEN deficiency promotes macrophage infiltration and hypersensitivity of prostate cancer to IAP antagonist/radiation combination therapy. Oncotarget 7 7885-7898. (https://doi. org/10.18632/oncotarget.6955)

Bansal D, Reimers MA, Knoche EM \& Pachynski RK 2021 Immunotherapy and immunotherapy combinations in metastatic castration-resistant prostate cancer. Cancers 13 334. (https://doi.org/10.3390/ cancers13020334)

Beer TM, Kwon ED, Drake CG, Fizazi K, Logothetis C, Gravis G, Ganju V, Polikoff J, Saad F, Humanski P, et al. 2017 Randomized, double-blind, phase III trial of ipilimumab versus placebo in asymptomatic or minimally symptomatic patients with metastatic chemotherapy-naive castration-resistant prostate cancer. Journal of Clinical Oncology 35 40-47. (https://doi.org/10.1200/JCO.2016.69.1584)

Ben-Batalla I, Vargas-Delgado ME, von Amsberg G, Janning M \& Loges S 2020 Influence of androgens on immunity to self and foreign: effects on immunity and cancer. Frontiers in Immunology 11 1184-1184. (https://doi.org/10.3389/fimmu.2020.01184)

Bezzi M, Seitzer N, Ishikawa T, Reschke M, Chen M, Wang G, Mitchell C, Ng C, Katon J, Lunardi A, et al. 2018 Diverse genetic-driven immune landscapes dictate tumor progression through distinct mechanisms. Nature Medicine 24 165-175. (https://doi.org/10.1038/nm.4463)

Blattner M, Liu D, Robinson BD, Huang D, Poliakov A, Gao D, Nataraj S, Deonarine LD, Augello MA, Sailer V, et al. 2017 SPOP mutation drives prostate tumorigenesis in vivo through coordinate regulation of PI3K/ mTOR and AR signaling. Cancer Cell 31 436-451. (https://doi. org/10.1016/j.ccell.2017.02.004)

Bruni D, Angell HK \& Galon J 2020 The immune contexture and immunoscore in cancer prognosis and therapeutic efficacy. Nature Reviews: Cancer 20 662-680. (https://doi.org/10.1038/s41568-0200285-7)

Calagua C, Ficial M, Jansen CS, Hirz T, Del Balzo L, Wilkinson S, Lake R, Ku AT, Voznesensky O, Sykes DB, et al. 2021 A subset of localized prostate cancer displays an immunogenic phenotype associated with losses of key tumor suppressor genes. Clinical Cancer Research [epub]. (https://doi.org/10.1158/1078-0432.ccr-21-0121)

Calcinotto A, Spataro C, Zagato E, Di Mitri D, Gil V, Crespo M, De Bernardis G, Losa M, Mirenda M, Pasquini E, et al. 2018 IL-23 secreted by myeloid cells drives castration-resistant prostate cancer. Nature $\mathbf{5 5 9}$ 363-369. (https://doi.org/10.1038/s41586-018-0266-0)

Casbon AJ, Reynaud D, Park C, Khuc E, Gan DD, Schepers K, Passegué E \& Werb Z 2015 Invasive breast cancer reprograms early myeloid differentiation in the bone marrow to generate immunosuppressive neutrophils. PNAS 112 E566-E575. (https://doi.org/10.1073/ pnas.1424927112)

Chalabi M, Fanchi LF, Dijkstra KK, Van den Berg JG, Aalbers AG, Sikorska K, Lopez-Yurda M, Grootscholten C, Beets GL, Snaebjornsson P, et al. 2020 Neoadjuvant immunotherapy leads to pathological responses in MMRproficient and MMR-deficient early-stage colon cancers. Nature Medicine 26 566-576. (https://doi.org/10.1038/s41591-020-0805-8)

Chen Z, Trotman LC, Shaffer D, Lin HK, Dotan ZA, Niki M, Koutcher JA, Scher HI, Ludwig T, Gerald W, et al. 2005 Crucial role of P53dependent cellular senescence in suppression of PTEN-deficient tumorigenesis. Nature 436 725-730. (https://doi.org/10.1038/ nature03918)

Comito G, Giannoni E, Segura CP, Barcellos-de-Souza P, Raspollini MR, Baroni G, Lanciotti M, Serni S \& Chiarugi P 2014 Cancer-associated fibroblasts and M2-polarized macrophages synergize during prostate carcinoma progression. Oncogene 33 2423-2431. (https://doi. org/10.1038/onc.2013.191)

Conley-LaComb MK, Saliganan A, Kandagatla P, Chen YQ, Cher ML \& Chinni SR 2013 PTEN loss mediated AKT activation promotes prostate tumor growth and metastasis via CXCL12/CXCR4 signaling. Molecular Cancer 12 85. (https://doi.org/10.1186/1476-4598-12-85)

Conti DV, Darst BF, Moss LC, Saunders EJ, Sheng X, Chou A, Schumacher FR, Olama AAA, Benlloch S, Dadaev T, et al. 2021 Trans- ancestry genome-wide association meta-analysis of prostate cancer identifies new susceptibility loci and informs genetic risk prediction. Nature Genetics 53 65-75. (https://doi.org/10.1038/s41588-020-00748-0)

Costanzo-Garvey DL, Keeley T, Case AJ, Watson GF, Alsamraae M, Yu Y, Su K, Heim CE, Kielian T, Morrissey C, et al. 2020 Neutrophils are mediators of metastatic prostate cancer progression in bone. Cancer Immunology, Immunotherapy 69 1113-1130. (https://doi.org/10.1007/ s00262-020-02527-6)

de Bono JS, Guo C, Gurel B, De Marzo AM, Sfanos KS, Mani RS, Gil J, Drake CG \& Alimonti A 2020 Prostate carcinogenesis: inflammatory storms. Nature Reviews: Cancer 20 455-469. (https://doi.org/10.1038/ s41568-020-0267-9)

Desai K, McManus JM \& Sharifi N 2021 Hormonal therapy for prostate cancer. Endocrine Reviews 42 354-373. (https://doi.org/10.1210/endrev/ bnab002)

Di Mitri D, Toso A, Chen JJ, Sarti M, Pinton S, Jost TR, D’Antuono R, Montani E, Garcia-Escudero R, Guccini I, et al. 2014 Tumourinfiltrating GR-1+ myeloid cells antagonize senescence in cancer. Nature 515 134-137. (https://doi.org/10.1038/nature13638)

Di Mitri D, Mirenda M, Vasilevska J, Calcinotto A, Delaleu N, Revandkar A, Gil V, Boysen G, Losa M, Mosole S, et al. 2019 Re-education of tumorassociated macrophages by CXCR2 blockade drives senescence and tumor inhibition in advanced prostate cancer. Cell Reports 282156 2168.e5. (https://doi.org/10.1016/j.celrep.2019.07.068)

Fay EK \& Graff JN 2020 Immunotherapy in prostate cancer. Cancers 12 1752. (https://doi.org/10.3390/cancers12071752).

Fridlender ZG, Sun J, Kim S, Kapoor V, Cheng G, Ling L, Worthen GS \& Albelda SM 2009 Polarization of tumor-associated neutrophil phenotype by TGF- $\beta$ : 'N1' versus 'N2' TAN. Cancer Cell 16 183-194. (https://doi.org/10.1016/j.ccr.2009.06.017)

Gamat M \& McNeel DG 2017 Androgen deprivation and immunotherapy for the treatment of prostate cancer. Endocrine-Related Cancer 24 T297-T310. (https://doi.org/10.1530/ERC-17-0145)

Graff JN, Liang LW, Kim J \& Stenzl A 2021 KEYNOTE-641: phase III study of pembrolizumab plus enzalutamide for metastatic castrationresistant prostate cancer. Future Oncology [epub]. (https://doi. org/10.2217/fon-2020-1008)

Graham LS, Montgomery B, Cheng HH, Yu EY, Nelson PS, Pritchard C, Erickson S, Alva A \& Schweizer MT 2020 Mismatch repair deficiency in metastatic prostate cancer: response to PD-1 blockade and standard therapies. PLoS ONE 15 e0233260. (https://doi.org/10.1371/journal. pone.0233260)

Halabi S, Kelly WK, Ma H, Zhou H, Solomon NC, Fizazi K, Tangen CM, Rosenthal M, Petrylak DP, Hussain M, et al. 2016 Meta-analysis evaluating the impact of site of metastasis on overall survival in men with castration-resistant prostate cancer. Journal of Clinical Oncology 34 1652-1659. (https://doi.org/10.1200/JCO.2015.65.7270)

Hawley J, Obradovic A, Guo XV, Chaimowitz M, Easterlin CA, Dallos M, Lim EA, Drake CG \& Stein MN 2020 Dissecting the immune infiltrate induced by ADT and PD-1 blockade in men with metastatic hormonesensitive prostate cancer using single-cell RNA-sequencing. Journal of Clinical Oncology 38 (Supplement 15) e17548. (https://doi.org/10.1200/ JCO.2020.38.15_suppl.e17548)

He MX, Cuoco MS, Crowdis J, Bosma-Moody A, Zhang Z, Bi K, Kanodia A, Su MJ, Ku SY, Garcia MM, et al. 2021 Transcriptional mediators of treatment resistance in lethal prostate cancer. Nature Medicine 27 426-433. (https://doi.org/10.1038/s41591-021-01244-6).

Hinshaw DC \& Shevde LA 2019 The tumor microenvironment innately modulates cancer progression. Cancer Research 79 4557-4566. (https:// doi.org/10.1158/0008-5472.CAN-18-3962)

Jachetti E, Cancila V, Rigoni A, Bongiovanni L, Cappetti B, Belmonte B, Enriquez C, Casalini P, Ostano P, Frossi B, et al. 2018 Cross-talk between myeloid-derived suppressor cells and mast cells mediates tumor-specific immunosuppression in prostate cancer. Cancer Immunology Research 6 552-565. (https://doi.org/10.1158/2326-6066. CIR-17-0385)

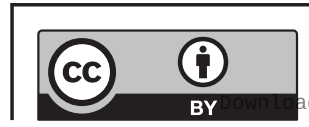

This work is licensed under a Creative Commons Attribution 4.0 International License. 
Jayaprakash P, Ai M, Liu A, Budhani P, Bartkowiak T, Sheng J, Ager C, Nicholas C, Jaiswal AR, Sun Y, et al. 2018 Targeted hypoxia reduction restores $\mathrm{T}$ cell infiltration and sensitizes prostate cancer to immunotherapy. Journal of Clinical Investigation 128 5137-5149. (https://doi.org/10.1172/JCI96268)

Jenzer M, Keß P, Nientiedt C, Endris V, Kippenberger M, Leichsenring J, Stögbauer F, Haimes J, Mishkin S, Kudlow B, et al. 2019 The BRCA2 mutation status shapes the immune phenotype of prostate cancer. Cancer Immunology, Immunotherapy 68 1621-1633. (https://doi. org/10.1007/s00262-019-02393-x)

Jiao S, Subudhi SK, Aparicio A, Ge Z, Guan B, Miura Y \& Sharma P 2019 Differences in tumor microenvironment dictate $\mathrm{T}$ helper lineage polarization and response to immune checkpoint therapy. Cell 179 1177.e13-1190.e13. (https://doi.org/10.1016/j.cell.2019.10.029)

Kantoff PW, Higano CS, Shore ND, Berger ER, Small EJ, Penson DF, Redfern CH, Ferrari AC, Dreicer R, Sims RB, et al. 2010 Sipuleucel-T immunotherapy for castration-resistant prostate cancer. New England Journal of Medicine 363 411-422. (https://doi.org/10.1056/ NEJMoa1001294)

Karzai F, VanderWeele D, Madan RA, Owens H, Cordes LM, Hankin A, Couvillon A, Nichols E, Bilusic M, Beshiri ML, et al. 2018 Activity of durvalumab plus olaparib in metastatic castration-resistant prostate cancer in men with and without DNA damage repair mutations. Journal for ImmunoTherapy of Cancer 6 141-141. (https://doi. org/10.1186/s40425-018-0463-2)

Kaur HB, Guedes LB, Lu J, Maldonado L, Reitz L, Barber JR, De Marzo AM, Tosoian JJ, Tomlins SA, Schaeffer EM, et al. 2018 Association of tumorinfiltrating T-cell density with molecular subtype, racial ancestry and clinical outcomes in prostate cancer. Modern Pathology 31 1539-1552. (https://doi.org/10.1038/s41379-018-0083-x)

Kwon ED, Drake CG, Scher HI, Fizazi K, Bossi A, van den Eertwegh AJM, Krainer M, Houede N, Santos R, Mahammedi H, et al. 2014 Ipilimumab versus placebo after radiotherapy in patients with metastatic castration-resistant prostate cancer that had progressed after docetaxel chemotherapy (CA184-043): a multicentre, randomised, double-blind, phase 3 trial. Lancet: Oncology 15 700-712. (https://doi.org/10.1016/ S1470-2045(14)70189-5)

Li S, Zhu M, Pan R, Fang T, Cao YY, Chen S, Zhao X, Lei CQ, Guo L, Chen Y, et al. 2016 The tumor suppressor PTEN has a critical role in antiviral innate immunity. Nature Immunology 17 241-249. (https:// doi.org/10.1038/ni.3311)

Li L, Ameri AH, Wang S, Jansson KH, Casey OM, Yang Q, Beshiri ML, Fang L, Lake RG, Agarwal S, et al. 2019 EGR1 regulates angiogenic and osteoclastogenic factors in prostate cancer and promotes metastasis. Oncogene 38 6241-6255. (https://doi.org/10.1038/s41388-019-0873-8)

Loi S, Drubay D, Adams S, Pruneri G, Francis PA, Lacroix-Triki M, Joensuu H, Dieci MV, Badve S, Demaria S, et al. 2019 Tumor-infiltrating lymphocytes and prognosis: a pooled individual patient analysis of early-stage triple-negative breast cancers. Journal of Clinical Oncology 37 559-569. (https://doi.org/10.1200/JCO.18.01010)

Long X, Hou H, Wang X, Liu S, Diao T, Lai S, Hu M, Zhang S, Liu M \& Zhang H 2020 Immune signature driven by ADT-induced immune microenvironment remodeling in prostate cancer is correlated with recurrence-free survival and immune infiltration. Cell Death and Disease 11 779. (https://doi.org/10.1038/s41419-020-02973-1)

Lu X, Horner JW, Paul E, Shang X, Troncoso P, Deng P, Jiang S, Chang Q, Spring DJ, Sharma P, et al. 2017 Effective combinatorial immunotherapy for castration-resistant prostate cancer. Nature $\mathbf{5 4 3}$ 728-732. (https://doi.org/10.1038/nature21676)

Marcus L, Lemery SJ, Keegan P \& Pazdur R 2019 FDA approval summary: pembrolizumab for the treatment of microsatellite instability-high solid tumors. Clinical Cancer Research 25 3753-3758. (https://doi. org/10.1158/1078-0432.CCR-18-4070)

Mariathasan S, Turley SJ, Nickles D, Castiglioni A, Yuen K, Wang Y, Kadel EE, Koeppen H, Astarita JL, Cubas R, et al. 2018 TGF $\beta$ attenuates tumour response to PD-L1 blockade by contributing to exclusion of T cells. Nature 554 544-548. (https://doi.org/10.1038/nature25501)

Mateo J, Porta N, Bianchini D, McGovern U, Elliott T, Jones R, Syndikus I, Ralph C, Jain S, Varughese M, et al. 2020 Olaparib in patients with metastatic castration-resistant prostate cancer with DNA repair gene aberrations (TOPARP-B): a multicentre, open-label, randomised, phase 2 trial. Lancet: Oncology 21 162-174. (https://doi.org/10.1016/S14702045(19)30684-9)

Maxwell PJ, Coulter J, Walker SM, McKechnie M, Neisen J, McCabe N, Kennedy RD, Salto-Tellez M, Albanese C \& Waugh DJJ 2013 Potentiation of inflammatory CXCL8 signalling sustains cell survival in PTEN-deficient prostate carcinoma. European Urology 64 177-188. (https://doi.org/10.1016/j.eururo.2012.08.032)

Maxwell PJ, Neisen J, Messenger J \& Waugh DJJ 2014 Tumor-derived CXCL8 signaling augments stroma-derived CCL2-promoted proliferation and CXCL12-mediated invasion of PTEN-deficient prostate cancer cells. Oncotarget 5 4895-4908. (https://doi.org/10.18632/oncotarget.2052)

Mlecnik B, Tosolini M, Kirilovsky A, Berger A, Bindea G, Meatchi T, Bruneval P, Trajanoski Z, Fridman WH, Pagès F, et al. 2011 Histopathologic-based prognostic factors of colorectal cancers are associated with the state of the local immune reaction. Journal of Clinical Oncology 29 610-618. (https://doi.org/10.1200/ JCO.2010.30.5425)

Motz GT, Santoro SP, Wang LP, Garrabrant T, Lastra RR, Hagemann IS, Lal P, Feldman MD, Benencia F \& Coukos G 2014 Tumor endothelium FASL establishes a selective immune barrier promoting tolerance in tumors. Nature Medicine 20 607-615. (https://doi.org/10.1038/ nm.3541)

Nava Rodrigues D, Rescigno P, Liu D, Yuan W, Carreira S, Lambros MB, Seed G, Mateo J, Riisnaes R, Mullane S, et al. 2018 Immunogenomic analyses associate immunological alterations with mismatch repair defects in prostate cancer. Journal of Clinical Investigation 128 4441-4453. (https://doi.org/10.1172/JCI121924)

Petitprez F, Fossati N, Vano Y, Freschi M, Becht E, Lucianò R, Calderaro J, Guédet T, Lacroix L, Rancoita PMV, et al. 2019 PD-L1 expression and CD8+ T-cell infiltrate are associated with clinical progression in patients with node-positive prostate cancer. European Urology Focus 5 192-196. (https://doi.org/10.1016/j.euf.2017.05.013)

Philippou Y, Sjoberg HT, Murphy E, Alyacoubi S, Jones KI, GordonWeeks AN, Phyu S, Parkes EE, Gillies McKenna W, Lamb AD, et al. 2020 Impacts of combining anti-PD-L1 immunotherapy and radiotherapy on the tumour immune microenvironment in a murine prostate cancer model. British Journal of Cancer 123 1089-1100. (https://doi.org/10.1038/s41416-020-0956-X)

Qi Z, Xu Z, Zhang L, Zou Y, Yan W, Li C, Liu N \& Wu H 2020 Overcoming resistance to immune checkpoint therapy in PTEN-null prostate cancer by sequential intermittent anti-PI3K $\alpha / \beta / \delta$ and anti-PD- 1 treatment. bioRxiv. (https://doi.org/ 10.1101/2020.10.17.343608)

Rescigno P, Gurel B, Pereira R, Crespo M, Rekowski J, Rediti M, Barrero M, Mateo J, Bianchini D, Messina C, et al. 2021 Characterizing CDK12mutated prostate cancers. Clinical Cancer Research 27 566-574. (https:// doi.org/10.1158/1078-0432.CCR-20-2371)

Robinson D, Van Allen E, Wu Y, Schultz N, Lonigro R, Mosquera J, Montgomery B, Taplin M, Pritchard C, Attard G, et al. 2015 Integrative clinical genomics of advanced prostate cancer. Cell 161 1215-1228. (https://doi.org/10.1016/j.cell.2015.05.001)

Shalapour S, Font-Burgada J, Di Caro G, Zhong Z, Sanchez-Lopez E, Dhar D, Willimsky G, Ammirante M, Strasner A, Hansel DE, et al. 2015 Immunosuppressive plasma cells impede T-cell-dependent immunogenic chemotherapy. Nature 521 94-98. (https://doi. org/10.1038/nature14395)

Sharma P, Pachynski RK, Narayan V, Flechon A, Gravis G, Galsky MD, Mahammedi H, Patnaik A, Subudhi SK, Ciprotti M, et al. 2019 Initial results from a phase II study of nivolumab (NIVO) plus ipilimumab (IPI) for the treatment of metastatic castration-resistant prostate cancer https://erc.bioscientifica.com https://doi.org/10.1530/ERC-21-0149 (c) 2021 The authors Published by Bioscientifica Ltd. Printed in Great Britain
This work is licensed under a Creative Commons Attribution 4.0 International License. ded from Bioscientifica.com at $04 / 26 / 2023$ 02:00:46PM 
(mCRPC; Checkmate 650). Journal of Clinical Oncology 37 (Supplement 7) 142-142. (https://doi.org/10.1200/JCO.2019.37.7_suppl.142)

Siegel RL, Miller KD \& Jemal A 2020 Cancer statistics, 2020. CA: A Cancer Journal for Clinicians 70 7-30. (https://doi.org/10.3322/caac.21590)

Sung H, Ferlay J, Siegel RL, Laversanne M, Soerjomataram I, Jemal A \& Bray F 2021 Global cancer statistics 2020: GLOBOCAN estimates of incidence and mortality worldwide for 36 cancers in 185 countries. CA: A Cancer Journal for Clinicians 71 209-249. (https://doi.org/ 10.3322/caac.21660)

Swami U, Isaacsson Velho P, Nussenzveig R, Chipman J, Sacristan Santos V, Erickson S, Dharmaraj D, Alva AS, Vaishampayan UN, Esther J, et al. 2020 Association of SPOP mutations with outcomes in men with de novo metastatic castration-sensitive prostate cancer. European Urology 78 652-656. (https://doi.org/10.1016/j.eururo.2020.06.033)

Sweeney CJ, Gillessen S, Rathkopf D, Matsubara N, Drake C, Fizazi K, Piulats JM, Wysocki PJ, Buchschacher GL, Doss J, et al. 2020 IMbassador250: a phase III trial comparing atezolizumab with enzalutamide vs enzalutamide alone in patients with metastatic castration-resistant prostate cancer (mCRPC). Cancer Research 80 abstract CT014. (https://doi.org/10.1158/1538-7445.AM2020-CT014)

Taylor RA, Fraser M, Livingstone J, Espiritu SMG, Thorne H, Huang V, Lo W, Shiah YJ, Yamaguchi TN, Sliwinski A, et al. 2017 Germline BRCA2 mutations drive prostate cancers with distinct evolutionary trajectories. Nature Communications 8 13671-13671. (https://doi. org/10.1038/ncomms13671).

Toso A, Revandkar A, Di Mitri D, Guccini I, Proietti M, Sarti M, Pinton S, Zhang J, Kalathur M, Civenni G, et al. 2014 Enhancing chemotherapy efficacy in PTEN-deficient prostate tumors by activating the senescence-associated antitumor immunity. Cell Reports 9 75-89. (https://doi.org/10.1016/j.celrep.2014.08.044).

Van Allen EM, Miao D, Schilling B, Shukla SA, Blank C, Zimmer L, Sucker A, Hillen U, Foppen MHG, Goldinger SM, et al. 2015 Genomic correlates of response to CTLA-4 blockade in metastatic melanoma. Science 350 207-211. (https://doi.org/10.1126/science.aad0095).

Vidotto T, Saggioro FP, Jamaspishvili T, Chesca DL, Picanço de Albuquerque CG, Reis RB, Graham CH, Berman DM, Siemens DR, Squire JA, et al. 2019 PTEN-deficient prostate cancer is associated with an immunosuppressive tumor microenvironment mediated by increased expression of IDO1 and infiltrating FoxP3+ T regulatory cells. Prostate 79 969-979. (https://doi.org/10.1002/pros.23808)
Vikas P, Borcherding N, Chennamadhavuni A \& Garje R 2020 Therapeutic potential of combining PARP inhibitor and immunotherapy in solid tumors. Frontiers in Oncology 10 570-570. (https://doi.org/10.3389/ fonc.2020.00570)

Weiner AB, Vidotto T, Liu Y, Mendes AA, Salles DC, Faisal FA, Murali S, McFarlane M, Imada EL, Zhao X, et al. 2021 Plasma cells are enriched in localized prostate cancer in black men and are associated with improved outcomes. Nature Communications 12 935. (https://doi. org/10.1038/s41467-021-21245-w)

Woo JR, Liss MA, Muldong MT, Palazzi K, Strasner A, Ammirante M, Varki N, Shabaik A, Howell S, Kane CJ, et al. 2014 Tumor infiltrating B-cells are increased in prostate cancer tissue. Journal of Translational Medicine 12 30. (https://doi.org/10.1186/1479-5876-12-30)

Wu Z, Chen H, Luo W, Zhang H, Li G, Zeng F \& Deng F 2020 The landscape of immune cells infiltrating in prostate cancer. Frontiers in Oncology 10 517637. (https://doi.org/10.3389/fonc.2020.517637)

Yang M, Liu J, Piao C, Shao J \& Du J 2015 ICAM-1 suppresses tumor metastasis by inhibiting macrophage M2 polarization through blockade of efferocytosis. Cell Death and Disease 6 e1780. (https://doi. org/10.1038/cddis.2015.144)

Zaanan A, Shi Q, Taieb J, Alberts SR, Meyers JP, Smyrk TC, Julie C, Zawadi A, Tabernero J, Mini E, et al. 2018 Role of deficient DNA mismatch repair status in patients with stage III colon cancer treated with FOLFOX adjuvant chemotherapy: a pooled analysis from 2 randomized clinical trials. JAMA Oncology 4 379-383. (https://doi. org/10.1001/jamaoncol.2017.2899)

Zhang J, Bu X, Wang H, Zhu Y, Geng Y, Nihira NT, Tan Y, Ci Y, Wu F, Dai X, et al. 2018 Cyclin D-CDK4 kinase destabilizes PD-L1 via cullin 3-SPOP to control cancer immune surveillance. Nature 553 91-95. (https://doi.org/10.1038/nature25015)

Zhao D, Lu X, Wang G, Lan Z, Liao W, Li J, Liang X, Chen JR, Shah S, Shang X, et al. 2017 Synthetic essentiality of chromatin remodelling factor chd1 in PTEN-deficient cancer. Nature 542 484-488. (https:// doi.org/10.1038/nature21357)

Zhao D, Cai L, Lu X, Liang X, Li J, Chen P, Ittmann M, Shang X, Jiang S, Li H, et al. 2020 Chromatin regulator chd1 remodels the immunosuppressive tumor microenvironment in PTEN-deficient prostate cancer. Cancer Discovery 10 1374-1387. (https://doi. org/10.1158/2159-8290.CD-19-1352)

Received in final form 10 June 2021

Accepted 15 June 2021

Accepted Manuscript published online 15 June 2021 (c) 2021 The authors Published by Bioscientifica Ltd. Printed in Great Britain

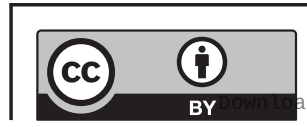

This work is licensed under a Creative Commons Attribution 4.0 International License. ded from Bioscientifica.com at 04/26/2023 02:00:46PM 